\title{
Study of Drug-Drug and Drug-Food Interactions of Mesalazine Through FTIR and DSC
}

\author{
Md. Mahbubul Alam ${ }^{1}$, Faria Tasneem ${ }^{2}$, A K Lutful Kabir ${ }^{1}$ \\ and Abu Shara Shamsur Rouf ${ }^{1}$
}

${ }^{1}$ Department of Pharmaceutical Technology, University of Dhaka, Dhaka-1000, Bangladesh

${ }^{2}$ Department of Pharmacy, University of Dhaka, Dhaka-1000, Bangladesh

(Received: March 16, 2019; Accepted: October 10, 2019; Published (web): December 19, 2019)

\begin{abstract}
A well-established drug used in the management of inflammatory bowel disease is 5-aminosalicylic acid (e.g. Mesalazine or Mesalamine). For the treatment of mild to moderate flares of ulcerative colitis and Crohn's disease, Mesalazine has been used as the first line drug in both western and Asian countries due to its superiority over other drugs in terms of side effects and toxicities. Besides, some other drugs are also prescribed for total resolution of different symptoms of ulcerative colitis and associated diseases, which include Acetaminophen, Metronidazole and Vitamin $\mathrm{D}_{3}$. Moreover, physicians instruct that Mesalazine should be taken at least one hour before meal. So, there are enough scopes of studying the drug-food interaction of Mesalazine to assess if there is any incompatibility present with food. Thus, in the present study, Mesalazine and physical mixtures of Mesalazine (1:1) in solid form along with the aforementioned drugs were prepared and analyzed to evaluate the compatibility among them using Fourier Transform Infrared Spectroscopy (FTIR) and Differential Scanning Calorimetry (DSC). In addition, interactions of Mesalazine with food stocks such as chicken and vegetable broth, fruit juice, milk and soybean oil were studied using FTIR, considering these as the common sources of protein, vitamin, fiber, minerals and fat. From this study, it was interpreted that, major interactions of Mesalazine were present with food samples. Besides, FTIR and DSC data revealed subtle clues of incompatibilities between Mesalazine and the other two drugs except Vitamin $\mathrm{D}_{3}$. So, the results may prove to be useful for related research works in future.
\end{abstract}

Key words: Mesalazine, interaction, compatibility, FTIR, DSC, ulcerative colitis, Crohn's disease

\section{INTRODUCTION}

Ulcerative colitis (UC) and Crohn's disease (CD) are chronic inflammatory bowel conditions, which are combinedly known as inflammatory bowel disease (IBD). The pathogenesis being still unknown, it is thought that this inflammatory process is peddled by an exaggerated immune response to antigenic stimulation by the gut microbiota on a background of genetic susceptibility.

In clinical practice, the disease activity of $\mathrm{CD}$ is typically described as mild to moderate (without manifestations of dehydration, abdominal tenderness or more than $10 \%$ weight loss), moderate to severe (more prominent symptoms such as fever, weight

Correspondence to: A K Lutful Kabir

Email: lutful@du.ac.bd

Dhaka Univ. J. Pharm. Sci. 18(2): 257-269, 2019 (December)

DOI: https://doi.org/10.3329/dujps.v18i2.44466 loss, abdominal pain, intermittent nausea and vomiting or significant anemia) and severe to fulminant disease (high fever, persisting vomiting or evidence of intestinal obstruction). Similar to CD, the severity of UC is classified as mild, moderate, severe and fulminant. ${ }^{1}$

Since the late 1970's, Mesalazine has been used as the gold standard first line treatment for IBD. It is an anti-inflammatory agent which is structurally related to the salicylates. Known as 5-aminosalicylic acid (5-ASA), it is monohydroxybenzoic acid substituted by an amino group at the $\mathrm{C}_{5}$-position and the functional groups present are significantly IR active. It is odorless and textured as white to pinkish crystals or purplish-tan powder. The aqueous solution is acidic and $\mathrm{pH}$ is approximately 4.1 at 0.8 
$\mathrm{mg} / \mathrm{l}$ water. Mesalazine is slightly soluble in water, alcohol and hydrochloric acid; more soluble in hot water. It may reduce inflammation through inhibition of cyclooxygenase and prostaglandin production. Following rectal or oral administration, only a small amount of Mesalazine is absorbed; the remainder acting topically, reduces diarrhea, rectal bleeding and stomach pain associated with IBD. ${ }^{2,3}$

The prevalence of Crohn's disease and ulcerative colitis has been on the rise in western countries and in the Asian countries as well. These are not uncommon in Bangladesh and are being diagnosed more commonly. On this view, the research work was accomplished by means of IR and DSC methods so as to establish a rapid, simple and precise interaction profile of Mesalazine with food and commonly prescribed drugs with it. ${ }^{4}$

\section{MATERIALS AND METHODS}

Chemicals and reagents. Analytical grade isopropyl alcohol (IPA) and acetone procured from RCI Labscan Ltd. (Thailand), were used to clean the FTIR machine and the required glass apparatus. Potassium dihydrogen phosphate and disodium hydrogen phosphate were purchased from BDH Chemicals (England). Acetaminophen, Metronidazole and dry Vitamin $\mathrm{D}_{3}$ were provided as generous gifts from Incepta Pharmaceuticals Limited and Mesalazine was obtained from Unimed and Unihealth Mfg. Ltd., Bangladesh. The chicken stock, vegetable stock and fruit juice were prepared at home. Soybean and milk were collected from a local market in Dhaka city. The drug samples were refrigerated under proper temperature.

Preparation of food stocks. Chicken stock was prepared by boiling bony chicken pieces in water along with bay leaf, cardamom, cinnamon, cloves, black cumin and garlic. Then the boiled water was used as the stock. Vegetable stock was prepared by boiling raw papaya, pointed gourd and snake gourd with a very small amount of mashed ginger in it. The boiled water was used as the stock. Fruit juice was prepared by squashing orange, grapes and with it, apple extract was added and filtered through
Whatman filter paper (No. 1) to get the juice in proper form.

Preparation of phosphate buffer of $\mathrm{pH}$ 7.4. Eudragit coated Mesalazine dissolves at intraluminal pH 6, and Eudragit S-coated Mesalazine works optimally at $\mathrm{pH} 7$ or above. So in this current experiment, we prepared a phosphate buffer solution of $\mathrm{pH} 7.4$ to evaluate the IR spectra of Mesalazine. Disodium hydrogen phosphate solution of $0.01 \mathrm{M}$ was prepared in a volumetric flask (solution A) and then, potassium dihydrogen phosphate solution of $0.02 \mathrm{M}$ was prepared in another volumetric flask (solution B), both measuring $500 \mathrm{ml}$. Afterwards, 290 $\mathrm{ml}$ of solution A was properly mixed with $66 \mathrm{ml}$ of solution B. Finally, the solution was made $1000 \mathrm{ml}$ by adding distilled water and $\mathrm{pH}$ was checked by a pH meter. ${ }^{5}$

In vitro IR studies of samples. IR spectra of the drugs Mesalazine, Acetaminophen, Metronidazole and dry Vitamin $\mathrm{D}_{3}$ were recorded in the solid state by putting them individually on the sample platform of the instrument. The bands were assigned in the range of $4000-600 \mathrm{~cm}^{-1}$. Background was checked before every analysis. The force gauge was kept at 60. In the similar way, spectrum data of mixture of Mesalazine with Acetaminophen, Metronidazole and dry Vitamin $\mathrm{D}_{3}$ each were taken by preparing a mixture of the drugs in the ratio $1: 1{ }^{6}$

Since Mesalazine works best at $\mathrm{pH} 7$ or above, the solution of the required $\mathrm{pH}$ was prepared in the lab and the IR data of the solution was taken individually. Afterwards, the IR spectrum of the solution in combination with Mesalazine was taken. In doing this, there required no force gauge and the liquid sample provided the necessary pressure to stay in contact with the crystal of the sample platform.

Individual IR data of soybean oil, vegetable and chicken stock, milk and fruit juice were obtained. Afterwards, data of the mixture of Mesalazine with the aforementioned foods were taken. These mixtures were made by blending Mesalazine (quantity sufficient) and the food samples in a petridish one at a time. 
In vitro DSC studies of samples. The sample Mesalazine was weighed and placed in an aluminum pan which was then hermetically sealed. Then it was put on a constantan disc on a platform in the DSC analysis cell with a chromel wafer below it. A chromel-alumel thermocouple under the constantan disc measured the sample temperature. An empty reference pan was put on a symmetric platform with its own underlying chromel wafer and chromelalumel thermocouple. Heat flow was determined by comparing the difference in temperature between the sample and the reference chromel wafers. The inert nitrogen gas flow rate was maintained at $20 \mathrm{ml} / \mathrm{min}$. Then from software, the thermogram of Mesalazine was obtained. Similar procedure was followed to get the thermograms of Acetaminophen, Metronidazole and dry Vitamin $\mathrm{D}_{3}$. The DSC data of physical mixtures of Mesalazine with Acetaminophen, Metronidazole and Vitamin $D_{3}$ in the ratio 1:1 each were also obtained. The DSC runs ranged from the temperature $30-400^{\circ} \mathrm{C}$ and the increase in temperature was $10^{\circ} \mathrm{C} / \mathrm{min}$.

\section{RESULTS AND DISCUSSION}

Fourier transform infrared spctroscopy (FTIR). The interactions between Mesalazine and drug and food samples were determined by using FTIR spectroscopy between the scan range of 4000$600 \mathrm{~cm}^{-1}$.

Compatibility study of Mesalazine. An IR spectra of pure Mesalazine illustrated the peaks of amine and carboxylic group because of the existence of amine (basic) and carboxylic acid (acidic) functional groups. The peaks could be considered as the characteristic peaks of Mesalazine (Table 1). ${ }^{7}$
These peaks were also presented by other researchers in their studies which were practically similar to the peaks found in our study, thereby indicating the identity and purity (Figures 4,5) of Mesalazine that we were working with. The IR spectra of Mesalazine showed no matching peaks with Acetaminophen, Metronidazole, Vitamin $\mathrm{D}_{3}$ or the food samples individually. On the contrary, the characteristic peaks of Mesalazine (2500.06, 1644.89, 1484.36, 1445.35, 1350.96, 806.18, 771.08 and $683.97 \mathrm{~cm}^{-1}$ ) were significantly observed in the IR spectra (Figures $4 \mathrm{~L}$, $4 \mathrm{M})$ of combination of Mesalazine with Metronidazole and dry Vitamin $\mathrm{D}_{3}$. However, substantial characteristic peaks of pure Mesalazine were absent in the spectra of combination of Mesalazine with the food samples such as soybean oil, milk, fresh fruit juice, vegetable broth and chicken broth.

From table 2, it was interpreted that, column (ii) showed the absence of three characteristic peaks of Mesalazine in the spectra of combination of Mesalazine and Acetaminophen in the fingerprint region, which might indicate the presence of possible interaction between them. The shift of the peaks might be attributed to the change in hydrogen bond strengths which, as a consequence, changed the vibrational frequency of the bands. ${ }^{9}$ Shift to higher wavelength value represented the reduction in bond length. Difference in intensities might be due to the difference of concentration of molecules in the samples. Again, the spectra of Mesalazine and combination of Mesalazine with Metronidazole in column (iii) and combination of Mesalazine with Vitamin $\mathrm{D}_{3}$ in column (iv) showed matching peaks,

Table 1. Characteristic peaks of Mesalazine. ${ }^{8}$

\begin{tabular}{lll}
\hline Peaks $\left(\mathrm{cm}^{-1}\right)$ & Range $\left(\mathrm{cm}^{-1}\right)$ & Functional Group \\
\hline 2500.06 & $3200-2500$ & Intramolecular hydrogen bonds, chelate compounds \\
1644.89 & $1650-1590$ & Primary amine $(\mathrm{N}-\mathrm{H}$ bend) \\
1484.36 & $1485-1445$ & C-H bending (alkane $\left.-\mathrm{CH}_{2}\right)$ \\
1445.35 & $1485-1445$ & C-H bending (alkane $\left.-\mathrm{CH}_{2}\right)$ \\
1350.96 & $1350-1260$ & O-H bending and C-O bending of primary or secondary alcohol \\
806.18 & $900-675$ & C-H stretch in aromatic compound \\
771.08 & $1000-700$ & C-H out of plane bending vibrations \\
683.97 & $900-670$ & Aromatic C-H out of plane bending vibrations \\
\hline
\end{tabular}



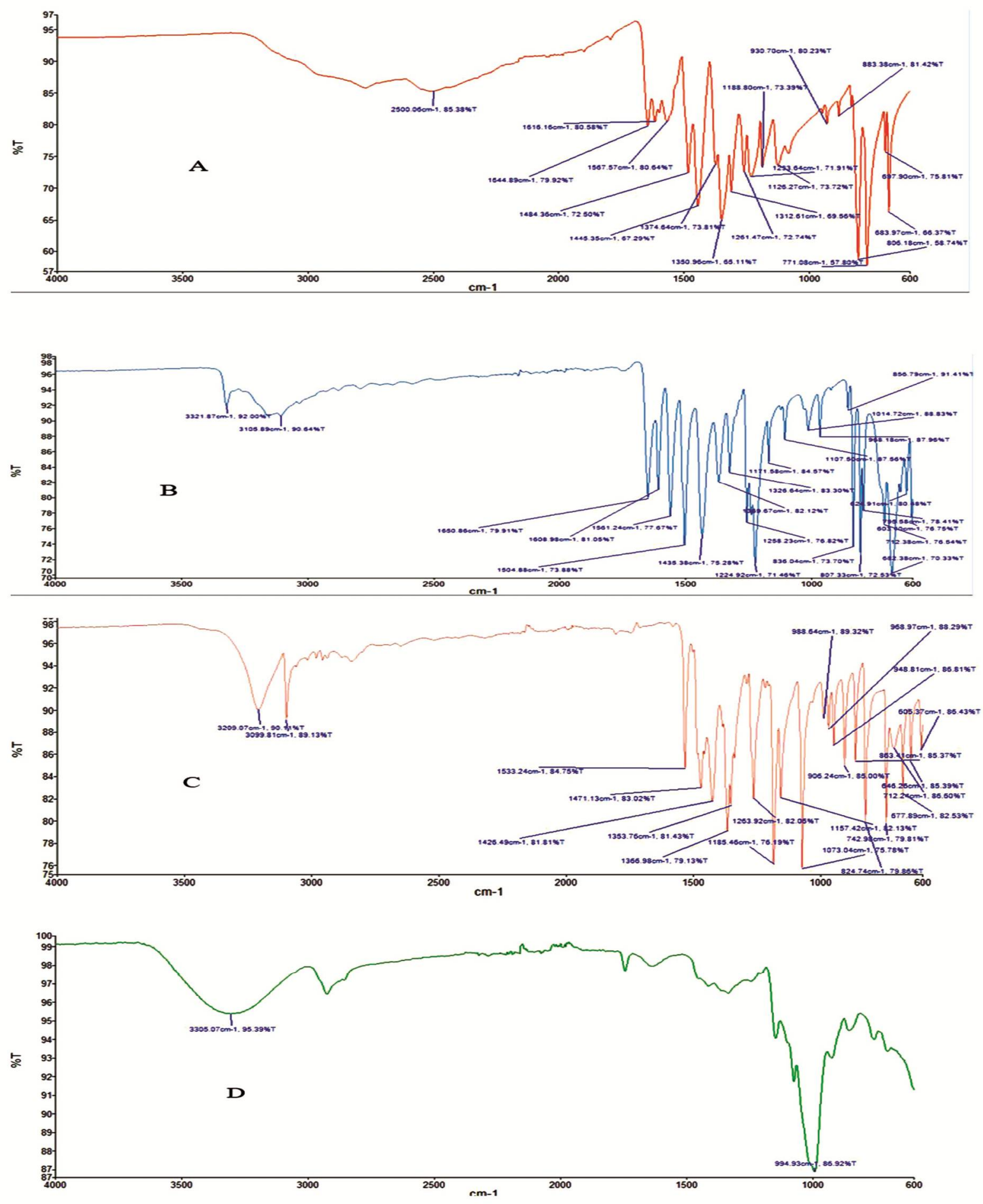

Figure 1. FTIR spectra of (A) Mesalazine, (B) Acetaminophen, (C) Metronidazole and (D) Vitamin $\mathrm{D}_{3}$ 

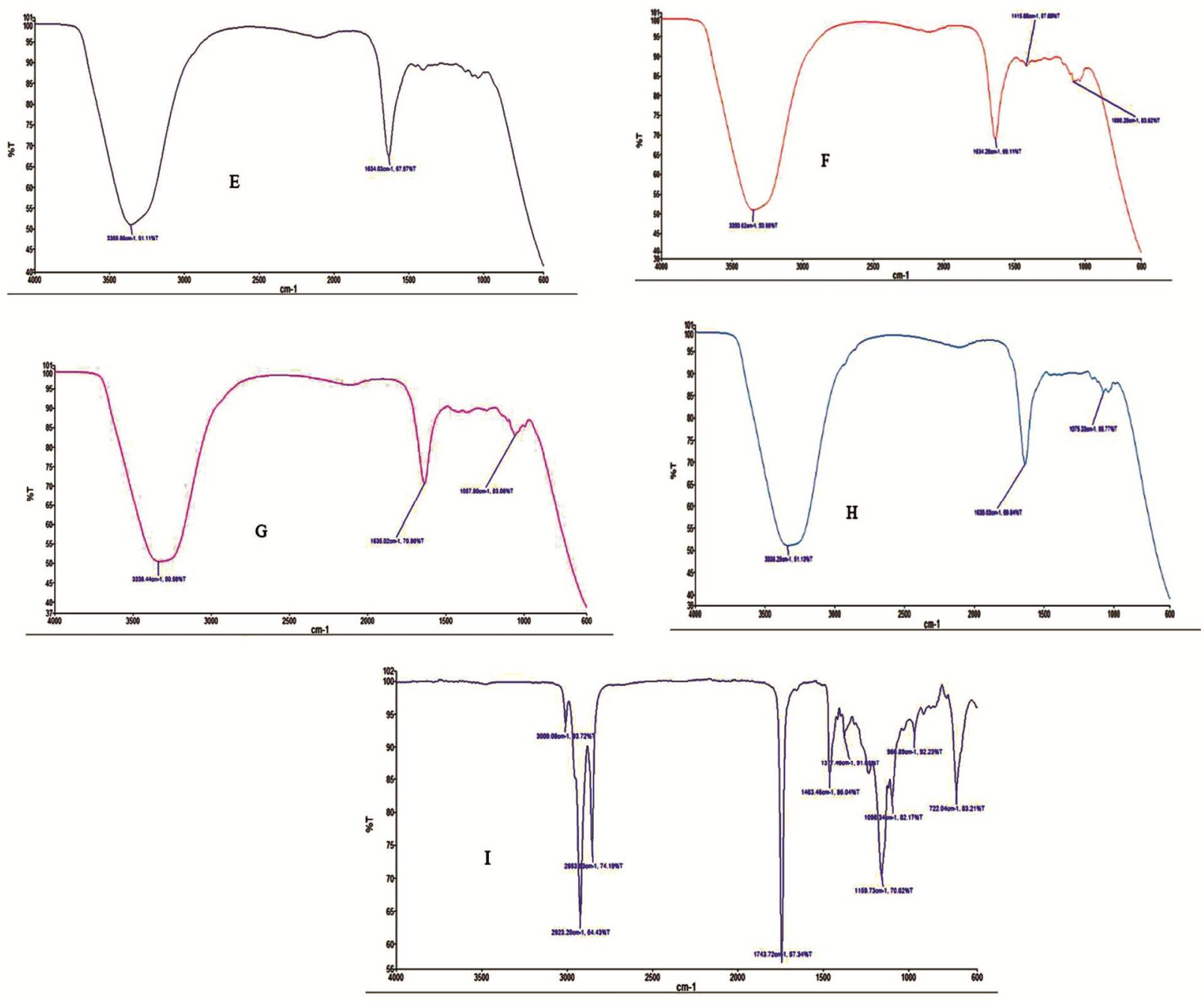

Figure 2. FTIR spectra of (E) Chicken stock, (F) Vegetable stock, (G) Fruit juice, (H) Milk and (I) Soybean oil.

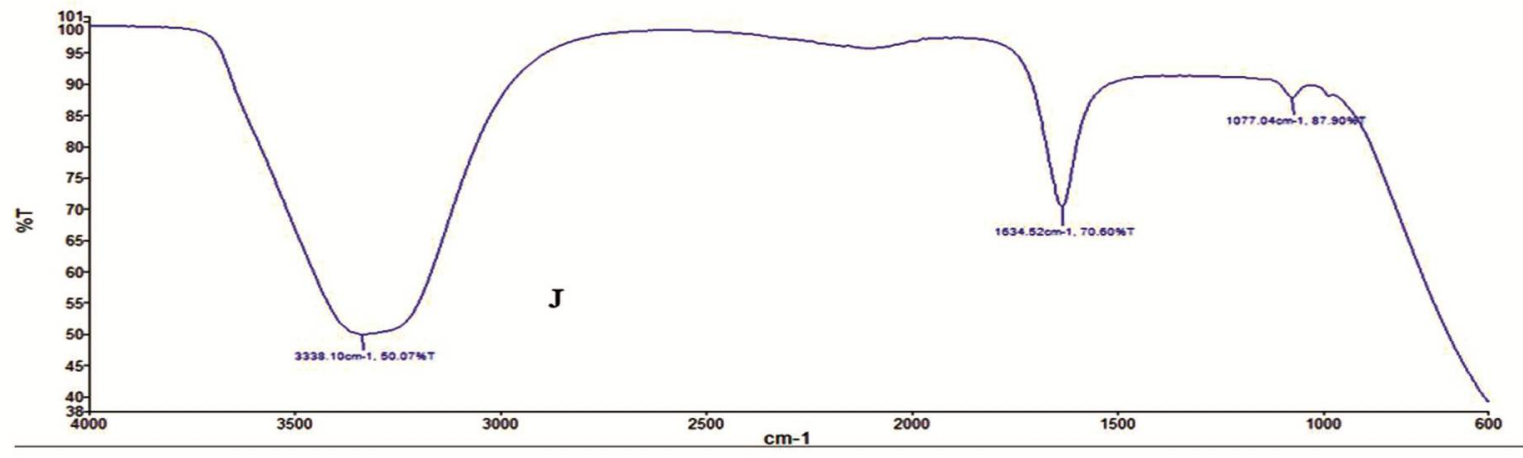

Figure 3. FTIR spectrum (J) of pH 7.4 buffer solution. 

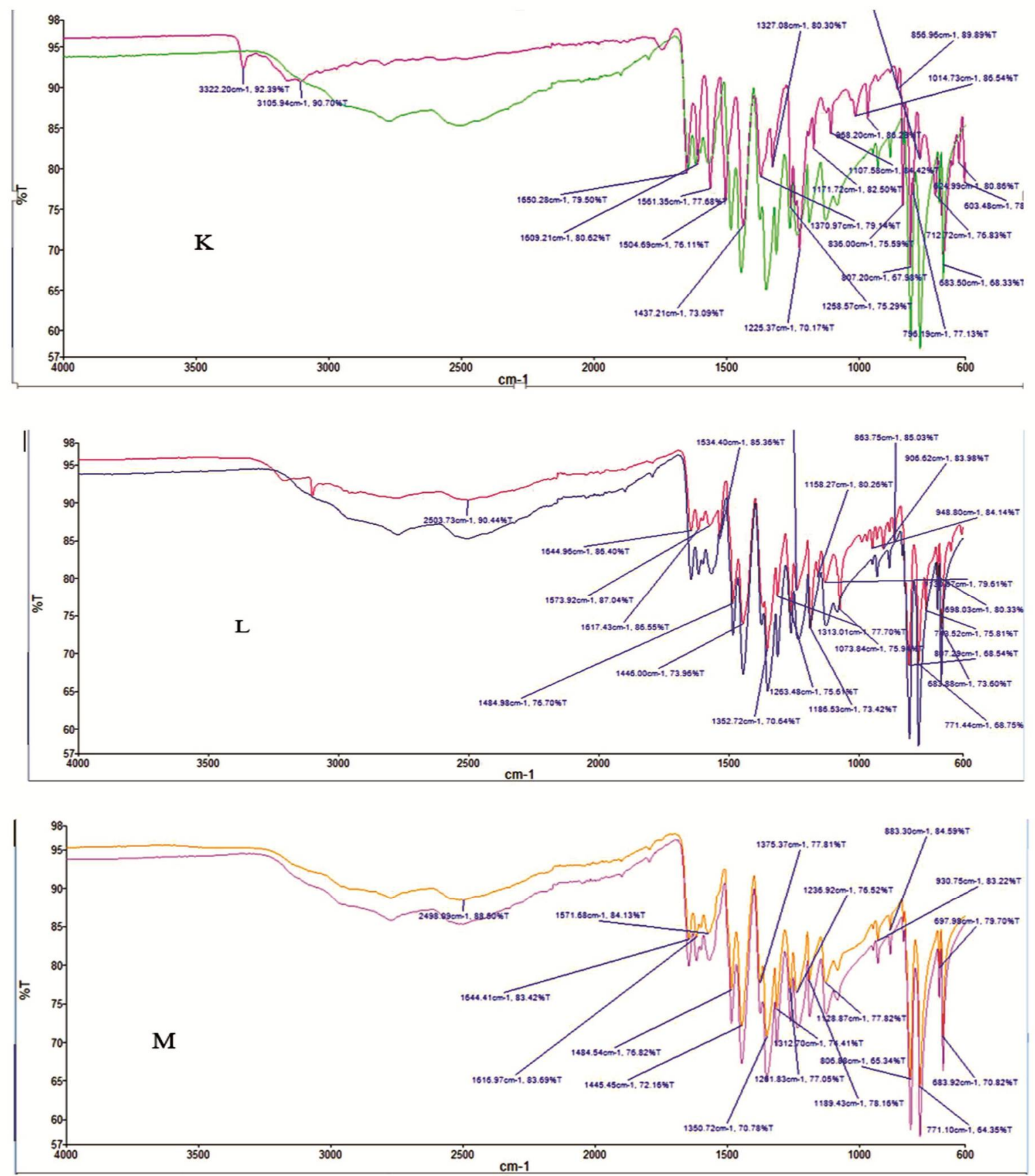

Figure 4. FTIR spectra of Mesalazine and (K) mixture of Mesalazine and Acetaminophen, (L) mixture of Mesalazine and Metronidazole and (M) mixture of Mesalazine and Vitamin $\mathrm{D}_{3}$. 


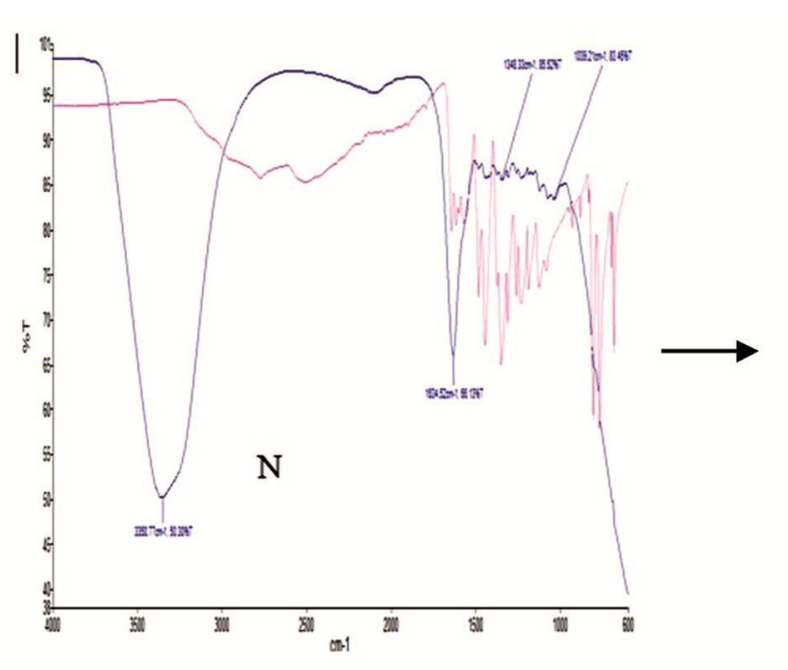

(a)

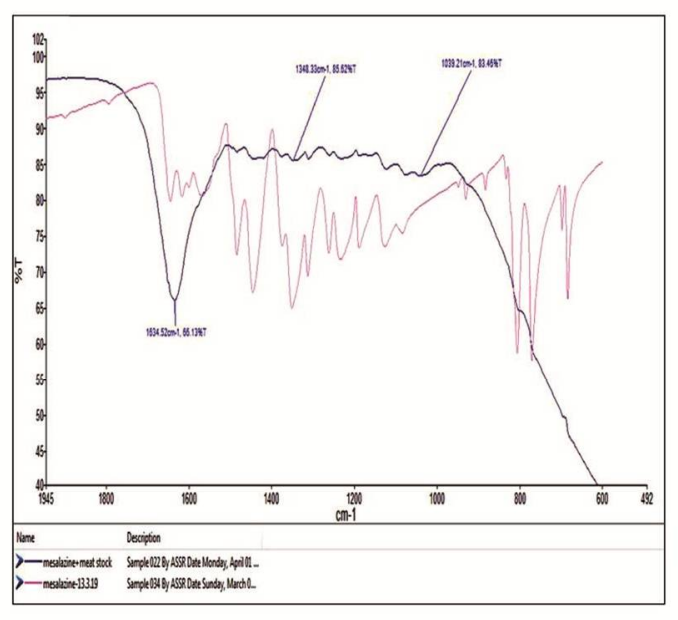

(b) Expanded spectrum of $\mathrm{N}$

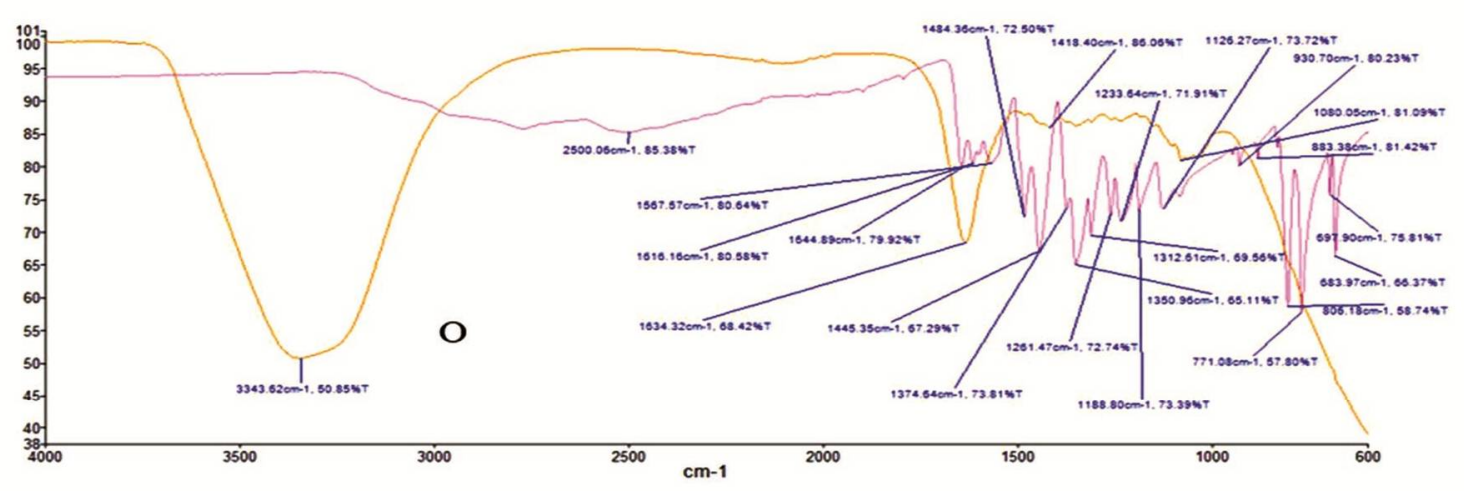

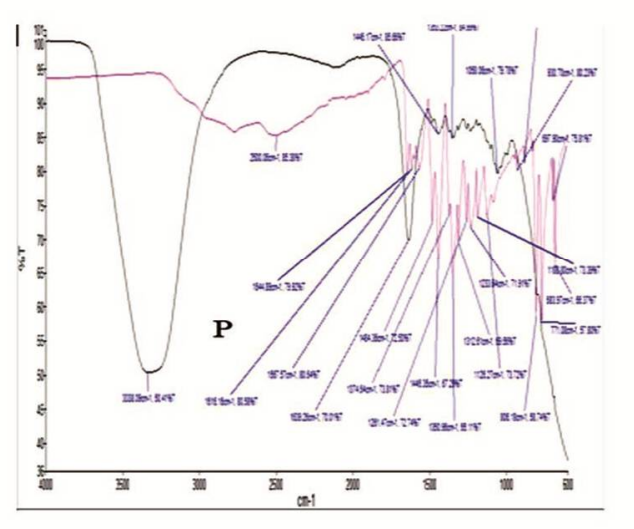

(a)

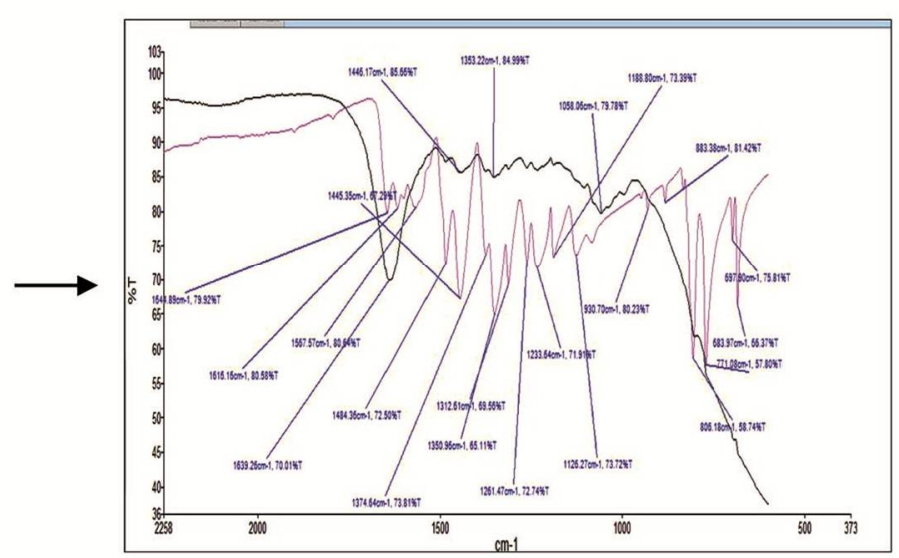

(b) Expanded spectrum of $\mathrm{P}$ 


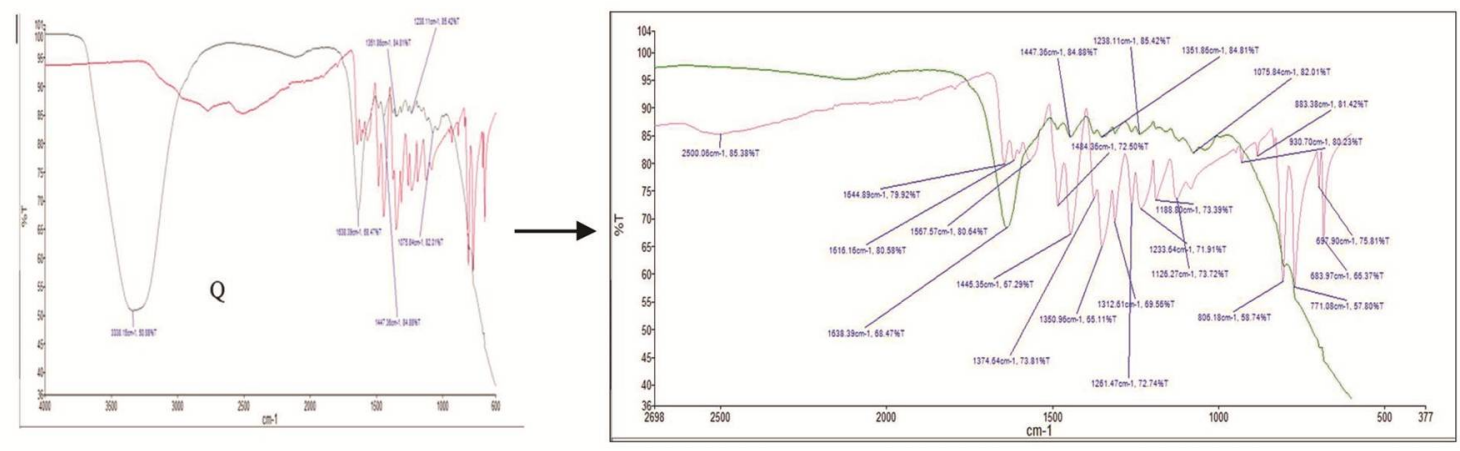

(a)

(b) Expanded spectrum of Q

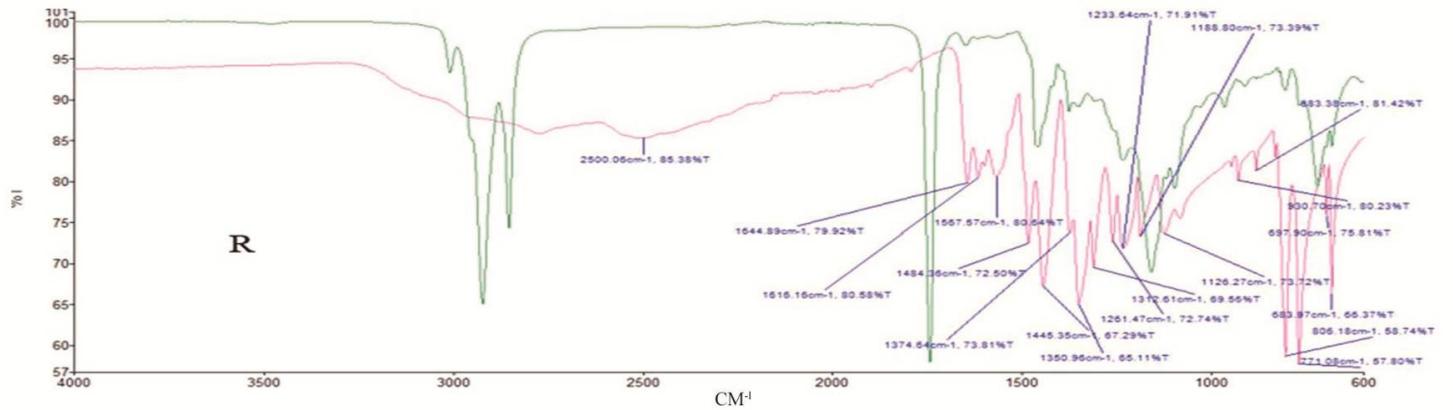

Figure 5. FTIR spectra of Mesalazine and (N) (a) mixture of Mesalazine and chicken stock, (b) close-up view of the fingerprint region with peak differences, $(\mathrm{O})$ mixture of Mesalazine and vegetable stock, (P) (a) mixture of Mesalazine and fruit juice, (b) close-up view of the fingerprint region with peak differences, (Q) (a) mixture of Mesalazine and milk, (b) close-up view of the fingerprint region with peak differences and (R) mixture of Mesalazine with soybean oil.

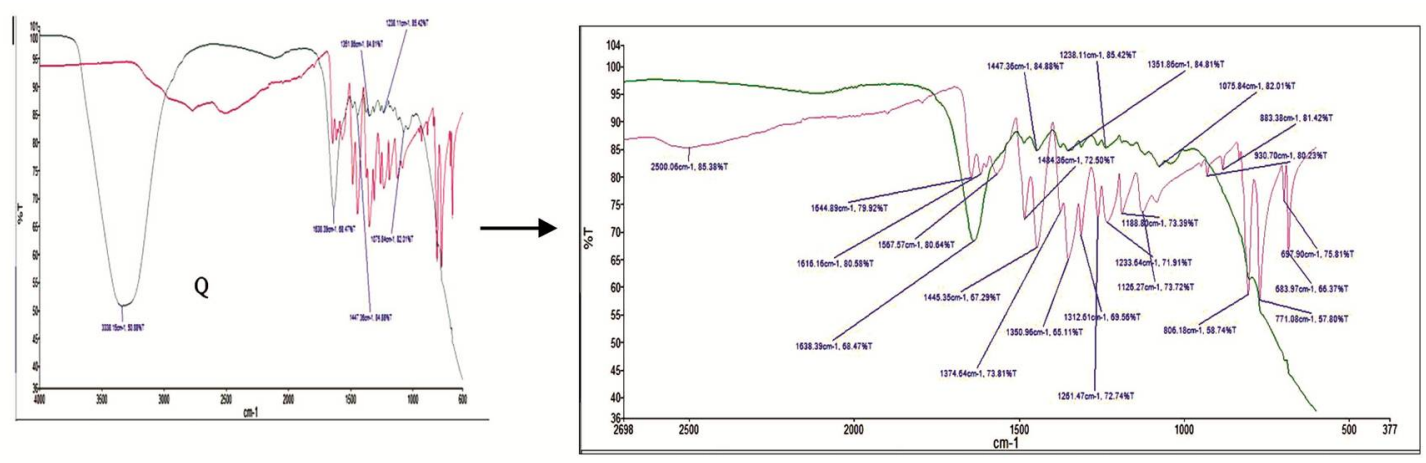

(a)

(b) Expanded spectrum of Q

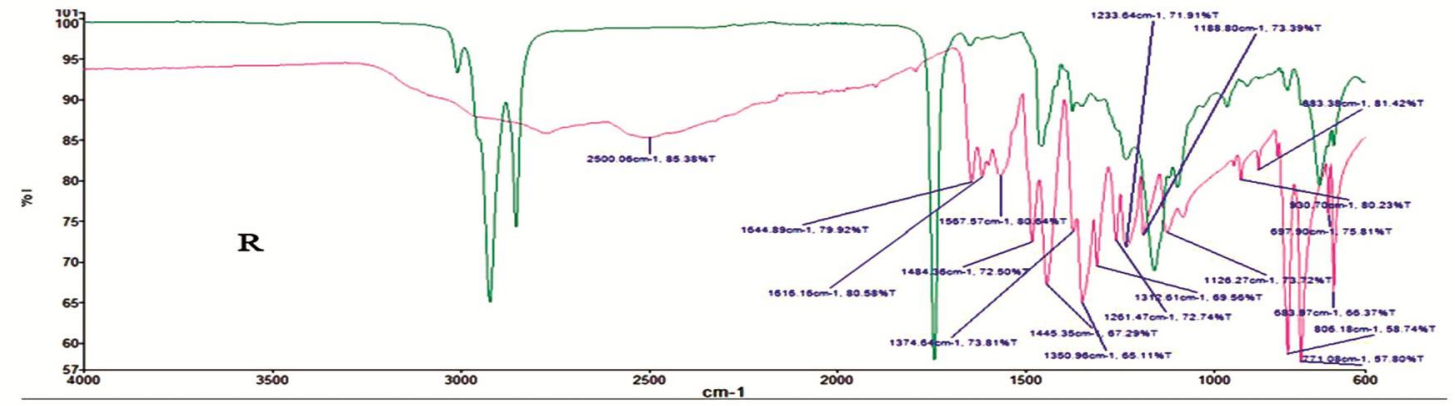

Figure 6. FTIR spectra of Mesalazine and (S) (a) mixture of Mesalazine and phosphate buffer of pH 7.4 and (b) close-up view of the fingerprint region with peak differences. 
Table 2. Comparison of characteristic peaks $\left(\right.$ in $^{\mathrm{cm}^{-1}}$ ) of mesalazine and combinations of mesalazine with drug and food samples.

\begin{tabular}{lccccccccc}
\hline $\begin{array}{l}\text { Peaks of } \\
\text { Mesalazine }\end{array}$ & $\begin{array}{c}\text { Peaks of } \\
\text { mixture of } \\
\text { Mesalazine } \\
\text { and } \\
\text { Acetamino- } \\
\text { phen }\end{array}$ & $\begin{array}{c}\text { Peaks of } \\
\text { mixture of } \\
\text { Mesalazine and } \\
\text { Metronidazole }\end{array}$ & $\begin{array}{c}\text { Peaks of } \\
\text { mixture of } \\
\text { Mesalazine } \\
\text { and dry } \\
\text { Vitamin } D_{3}\end{array}$ & $\begin{array}{c}\text { Peaks of } \\
\text { mixture of } \\
\text { Mesalazine } \\
\text { and chicken } \\
\text { stock }\end{array}$ & $\begin{array}{c}\text { Peaks of } \\
\text { mixture of } \\
\text { Mesalazine } \\
\text { and } \\
\text { vegetable } \\
\text { stock }\end{array}$ & $\begin{array}{c}\text { Peaks of } \\
\text { mixture of } \\
\text { Mesalazine } \\
\text { and fruit } \\
\text { juice }\end{array}$ & $\begin{array}{c}\text { Peaks of } \\
\text { mixture } \\
\text { of } \\
\text { Mesala- } \\
\text { zine and } \\
\text { milk }\end{array}$ & $\begin{array}{c}\text { Peaks of } \\
\text { mixture of } \\
\text { Mesalazine } \\
\text { and } \\
\text { soybean oil }\end{array}$ & $\begin{array}{c}\text { Peaks of } \\
\text { mixture of } \\
\text { Mesalazine } \\
\text { in pH 7.4 } \\
\text { solution }\end{array}$ \\
\hline (i) & (ii) & (iii) & (iv) & (v) & (vi) & (vii) & (viii) & (ix) & (x) \\
\hline 2500.06 & - & 2503.73 & 2498.09 & - & - & - & - & - & - \\
1644.89 & 1650.28 & 1644.96 & 1644.41 & - & - & - & - & - & 1644.76 \\
1484.36 & - & 1484.98 & 1484.54 & - & - & - & - & - & 1485.19 \\
1445.35 & 1437.21 & 1446.0 & 1445.45 & - & - & 1446.17 & 1447.36 & - & 1446.84 \\
1350.96 & - & 1352.72 & 1350.72 & 1348.33 & - & 1353.22 & 1351.86 & - & 1350.56 \\
806.18 & 807.2 & 807.29 & 806.88 & - & - & - & - & 806.25 & - \\
771.08 & 771.29 & 771.44 & 771.1 & - & - & - & - & - & - \\
683.97 & 683.5 & 683.88 & 683.92 & - & - & - & - & 684.13 & - \\
\hline
\end{tabular}

which marked the absence of any interaction of the drug with Metronidazole and Vitamin $\mathrm{D}_{3}$. However, the significant absence of characteristic peaks of Mesalazine in the spectra of combination of Mesalazine and chicken stock in column (v), Mesalazine and vegetable stock in column (vi), Mesalazine and fruit juice in column (vii), Mesalazine and milk in column and (viii) Mesalazine and soybean in column (ix) in the fingerprint region revealed the presence of possible interactions of the drug with foods. This also suggested that the properties of Mesalazine were also not retained, questioning its therapeutic advantage when taken simultaneously with the selected food items. Lastly, the absence of four characteristic peaks of Mesalazine in the spectra of combination of Mesalazine in buffer solution of $\mathrm{pH} 7.4$ in column (x) might suggest the presence of possible interactions between them, but from previously conducted studies by other researchers, it was found that the luminal release of 5-ASA from Mesalazine was possibly not repressed by the active inflammatory condition in IBD patients ${ }^{10}$ and worked satisfactorily to treat the flares of ulcerative colitis and Crohn's diseases. In addition, it can be perceived from the peaks that Mesalazine retained its own properties by keeping the amino functional group and carboxyl group undamaged and peaks that were absent did not take part in binding with the receptor sites, rather they only played role in securing the structural integrity of Mesalazine. Having been said this, we could assume that Mesalazine worked efficaciously in the $\mathrm{pH}$ above 7 , which is generally the intestinal milieu of IBD patients.

\section{Differential Scanning Calorimetry (DSC).}

The DSC thermograms of the individual drugs and the binary mixtures (1:1) of Mesalazine with Acetaminophen, Metronidazole and dry Vitamin $\mathrm{D}_{3}$ are shown in figure 7 and figure 8 respectively.

It can be easily found from figure 7 that the DSC curve of Mesalazine consisted of one endothermic peak at $286.21^{\circ} \mathrm{C}$, related to the melting process. In the same way, Acetaminophen showed one endothermic peak at $170.78^{\circ} \mathrm{C}$ and Vitamin D3 showed an exothermic peak at $197.37^{\circ}$ C. However, there was one sharp endothermic peaks of Metronidazole found at $164.75^{\circ} \mathrm{C}$.

In the DSC curve of the binary mixtures of other drugs with Mesalazine, the following events were seen.

The appearance of a peak in DSC curves of the mixtures (Mesalazine/ Metronidazole) and peak broadening and a shift towards lower temperature (Mesalazine/ Acetaminophen) owing to the melting deviations from characteristic peaks of the individual drugs suggested interactions indication. ${ }^{11}$ However, 

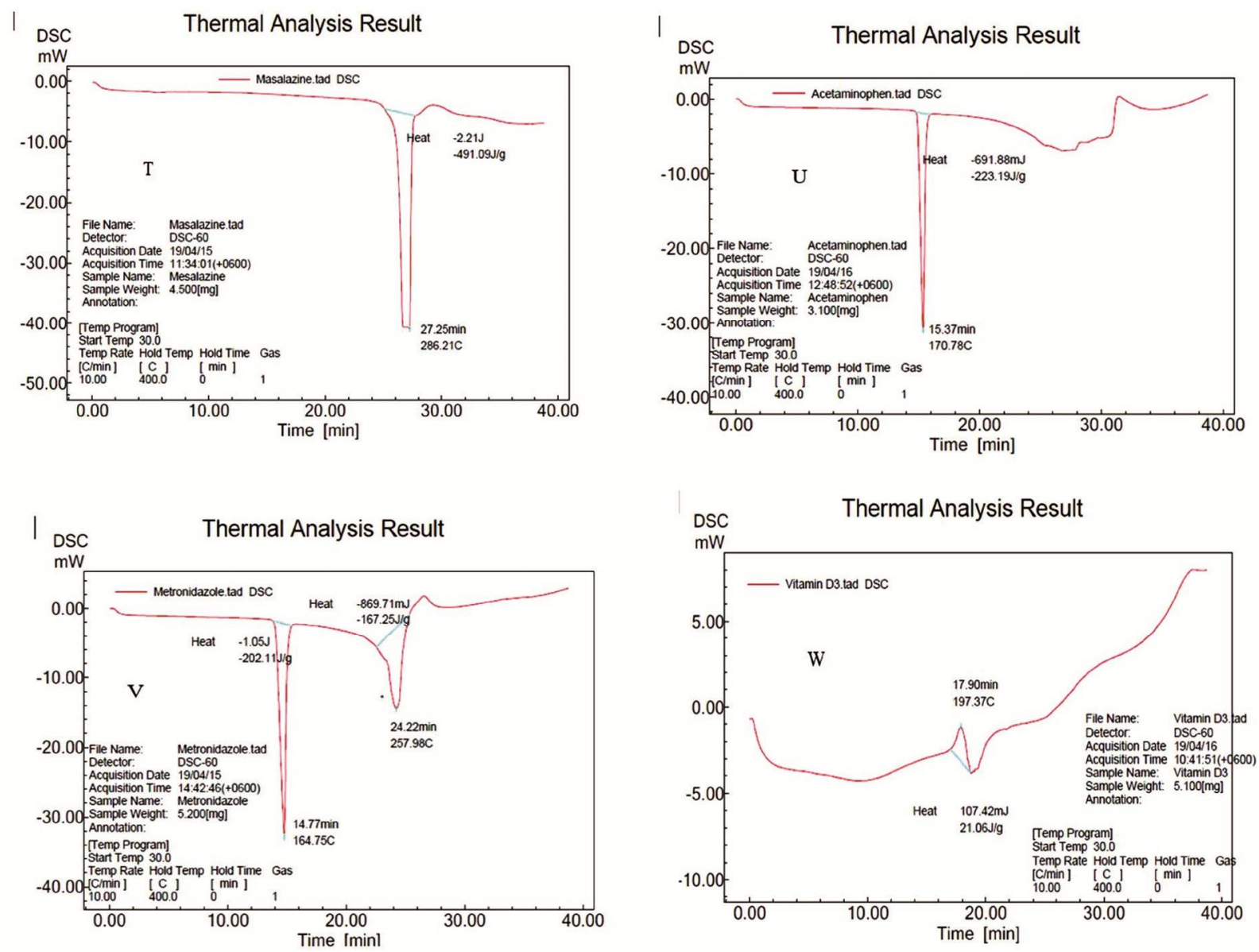

Figure 7. DSC thermograms of (T) Mesalazine, (U) Acetaminophen, (V) Metronidazole and (W) Vitamin $\mathrm{D}_{3}$.

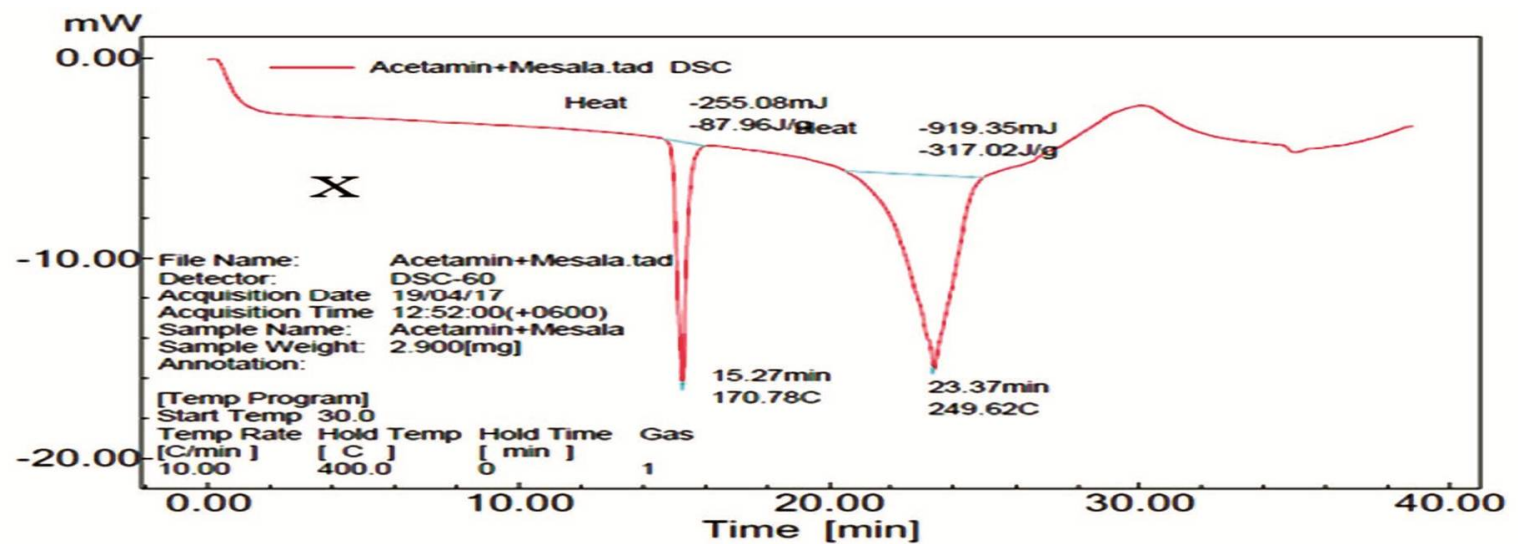



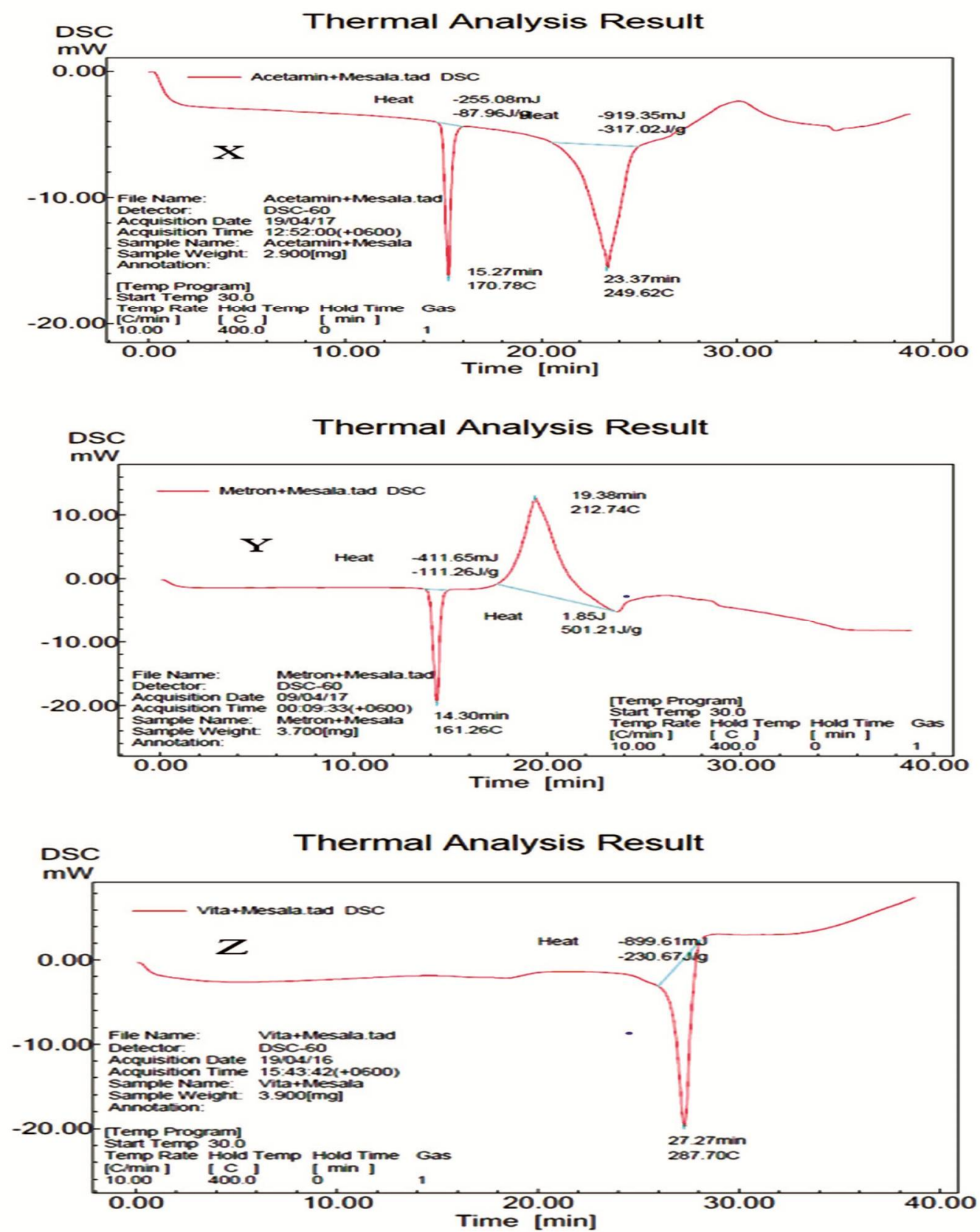

Figure 8. DSC thermograms of the 1:1 mixture of (X) Mesalazine and Acetaminophen, (Y) Mesalazine and Metronidazole and (Z) Mesalazine and Vitamin $\mathrm{D}_{3}$.

Table 3. Comparison of DSC data of Mesalazine and binary mixtures (1:1) of Mesalazine with other drug substances.

\begin{tabular}{lcccc}
\hline Sample & Endothermic Peak & Exothermic Peak & $\begin{array}{c}\text { Temperature } \\
\left({ }^{\circ} \mathrm{C}\right)\end{array}$ & $\begin{array}{c}\text { Peak considered for possible } \\
\text { interaction }\end{array}$ \\
\hline Mesalazine & Present & Absent & 286.21 & 286.21 \\
Mesalazine/ Acetaminophen & Present & Absent & 170.78 & 249.62 \\
& & & 249.62 & 212.74 \\
Mesalazine/ Metronidazole & Present & Present & 161.26 & 287.70 \\
Mesalazine/vit. D3 & & & 212.74 & 287.70 \\
\hline
\end{tabular}


the change in peaks might be obliged to the interactions between the drugs and the gas used in DSC, difference of molecular weights between the studied compounds or due to insufficient mixing when the binary combinations of the drugs were made. Also, very small particles in the mixture might amalgamate with larger particles causing surface interactions, but such physical interactions can not cause any hamper in the biosystem, because in the presence of aqueous gastrointestinal fluid, the smaller particles get separated from the larger particles to dissolve and make the drug bioavailable. ${ }^{12}$

\section{CONCLUSION}

The study was undertaken with an aim to evaluate the drug-drug and drug-food interactions of Mesalazine using the commonly prescribed drugs with it and various food samples. FTIR study of pure Mesalazine and its mixtures with other drug samples showed that there were no interactions of Mesalazine with Metronidazole and Vitamin $\mathrm{D}_{3}$, however, possible interactions may exist with Acetaminophen. On the other hand, from the FTIR study of Mesalazine and food samples, it was concluded that food shows major interactions with Mesalazine, if administered concurrently. So, it is better to avoid taking this medication with food.

Furthermore, the DSC study on the compatibility of Mesalazine with Acetaminophen, Metronidazole and dry Vitamin $D_{3}$ has shown that Vitamin $D_{3}$ fulfills its role as a compatible drug with Mesalazine, nonetheless, Acetaminophen and Metronidazole showed some clues of interactions, leaving us the space for further investigation with divergent analytical approaches.

Conclusively, it is worth mentioning that, Mesalazine may be prescribed with the above mentioned drugs concomitantly if the benefits outweigh the risks in specific patient groups but it should not be administered in conjunction with foods prerequisitely.

\section{ACKNOWLEDGEMENTS}

The authors are grateful to Higher Education Quality Enhancement Project (HEQEP), AIF, RoundIII, Window-2, CP-3245, Award No. 26, University Grants Commission (UGC), Bangladesh, for supporting the research work.

\section{AUTHORS' CONTRIBUTION}

Alam, M.M. conceived the presented idea. Alam, M.M. and Tasneem, F. developed the theory and performed the computations. Kabir, A.K.L. and Rouf, A.S.S. verified the analytical methods and supervised the findings of this work. All authors discussed the results and contributed to the final manuscript.

\section{CONFLICT OF INTEREST}

The authors declare that there was no conflict of interest.

\section{REFERENCES}

1. Iacucci, M., de Silva, S. and Ghosh, S. 2010. Mesalazine in inflammatory bowel disease: a trendy topic once again. Can. J. Gastroenter. 24, 127-133.

2. Bodegraven, A. 2006. Indications for 5-aminosalicylate in inflammatory bowel disease: is the body of evidence complete. World J. Gastroenter. 12, 6115.

3. Khan, A.K., Piris, J. and Truelove S.C. 1977. An experiment to determine the active therapeutic moiety of sulphasalazine. Lancet. 2, 892-895.

4. Bal, T. and Murthy, P.N. 2012. Int. J. Pharm. Sci. Res. 3, 561.

5. Perrin, D.D. and Dempsey, B. 1975. Buffers for $\mathrm{pH}$ and metal ion control. Anal. Chim. Acta. 78, 238.

6. Madni, A., Ekwal, M., Ahmad, S., Din, I., Hussain, Z., Ranjha, N.M., Khan, M.I., Akhlaq, M., Mahmood, M.A. and Zafar, H. 2014. FTIR drug-polymer interactions studies of perindopril erbumine. Chem. Soc. Pak. J. 36, 1064-1070.

7. Sandhya, P., Arshiya, H., Bhatnagar, D. and Poornima, P. 2013. Formulation and evaluation of colon specific drug delivery system of mesalamine. Int. J. of Biol. Pharm. Res. 4, 671-675. 
8. Coates, J. 1996. The interpretation of infrared spectra: published reference sources. Appl. Spectrosc. Rev. 31, 179192.

9. Ryu, S., Noda, I. and Jung, Y. 2010. What is the origin of positional fluctuation of spectral features: true frequency shift or relative intensity changes of two overlapped bands? Appl. Spectrosc. 64, 1017-1021.

10. Press, A.G., Hauptmann, I.A., Hauptmann, L., Fuchs, B., Fuchs, M., Ewe, K. and Ramadori, G. 1998. Gastrointestinal $\mathrm{pH}$ profiles in patients with inflammatory bowel disease. Aliment. Pharmacol. Ther. 12, 673-678.
11. Wesolowski, M. and Rojek, B. 2013. Thermogravimetric detection of incompatibilities between atenolol and excipients using multivariate techniques.J. Therm. Anal. Calorim. 113, 169-177.

12. Ainurofiq, A. and Choiri, S. 2017. The interaction of a binary/ternary interactive mixture of hydrophobichydrophilic materials on the drug distribution and drug release performance in the tablet formulation. IOP Conf. Ser.: Mater. Sci. Eng. 176, 120-126. 